\title{
Two simple, brief, naturalistic activities and their impact on positive affect: feeling grateful and eating ice cream
}

Peter Alex Linley ${ }^{1}$, Helen Dovey ${ }^{1}$, Eveline de Bruin ${ }^{2}$, Catherine Transler ${ }^{2}$, Joy Wilkinson ${ }^{3}$, John Maltby ${ }^{4}$ and Robert Hurling ${ }^{3^{*}}$

\author{
* Correspondence: \\ bob.hurling@unilever.com \\ ${ }^{3}$ Unilever Research, Colworth \\ Science Park, MK44 1LQ Bedford, UK \\ Full list of author information is \\ available at the end of the article
}

\begin{abstract}
Background: Positive psychology interventions have been shown to increase happiness and well-being, and researchers are beginning to speculate on the mechanisms through which these interventions may be effective. People are also naturally attuned to the things that will make them happier in their daily lives, and there is a case for considering how more naturalistic, everyday activities may also increase positive affect, happiness and well-being.

Methods: Study 1 involved 89 participants who completed a gratefulness activity while eating an ice cream for two days, following baseline measurement for two days. Participants completed the PANAS (Positive and Negative Affect Scale) twice per day, in the afternoon and in the evening, with the activity between these two times on the activity days. Study 2 involved 280 participants who formed four groups (gratefulness activity; ice cream consumption; gratefulness activity and ice cream consumption; control group), which undertook the relevant activity for two days. Participants completed baseline and follow-up measures, including the PANAS and the GQ-6 (Gratitude Questionnaire-6) on the day before and day following the two activity days. On the activity days themselves, participants completed the PANAS before and after their activity.
\end{abstract}

Results: In Study 1, we found that the combination of the gratefulness activity and eating ice cream led to increased positive affect. In Study 2, we explored this finding further, and found that any combination of the gratefulness activity and eating ice cream (together or alone) led to increased positive affect. The same pattern of results was observed for affect balance and the ratio of positive to negative emotions. The gratefulness activity only also led to increased gratitude as measured by the GQ-6. Results showed that even simple, naturalistic everyday activities can lead to increases in positive affect. This experience of positive affect may be one mediating pathway through which positive psychology interventions, and indeed everyday activities, are effective in enhancing happiness and well-being.

Conclusions: Researchers should consider the role of everyday activities in enhancing happiness and well-being, in addition to investigating the operational mechanisms of more formal positive psychology interventions.

Keywords: Positive affect; Gratefulness activity; Ice cream; Naturalistic activities

\section{Springer}

(c) 2013 Linley et al.; licensee Springer. This is an open access article distributed under the terms of the Creative Commons Attribution License (http://creativecommons.org/licenses/by/2.0), which permits unrestricted use, distribution, and reproduction in any medium, provided the original work is properly cited. 
The advent of positive psychology as a distinctive research discipline has led to a significantly increased focus on the psychology of well-being. It is well-established that people want to be happy, and to this end researchers have turned their attention to developing interventions that are designed to help people become happier.

Notwithstanding early happiness-increase researchers (e.g., Fordyce 1977), the prevailing view of much of psychology until the advent of positive psychology interventions had been that happiness is fleeting and ephemeral, and in any event, subject to the hedonic treadmill (Brickman \& Campbell 1971, but see also Diener et al. 2006). In recent years, however, the evidence is building that happiness can be increased sustainably (e.g., Sheldon \& Lyubomirsky 2006). With it now being more readily accepted that happiness can be increased, researchers have been turning their attention to understanding more about what works for whom, and how and why and when, in relation to positive psychology interventions (Layous \& Lyubomirsky in press).

Example positive psychology interventions reported to date include, amongst others, counting one's blessings (Emmons \& McCullough 2003), writing letters of gratitude (Boehm et al. 2011), writing about positive experiences (Burton \& King 2004), performing acts of kindness (Sheldon et al. in press), using signature strengths in new and different ways (Seligman et al. 2005), and writing about one's best possible self (Lyubomirsky et al. 2011).

Reviewing the effectiveness of positive psychology interventions, a meta-analysis of 51 studies by Sin and Lyubomirsky (2009) showed that they significantly enhanced well-being (mean $r=.29$ ) and also alleviated depression (mean $r=.31$ ). As such, there is clear evidence that positive psychology interventions work.

The mechanisms, by which they work, however, remain more open to investigation, and it is to this that researchers are now turning their attention. For example, Layous and Lyubomirsky (in press) have hypothesized four potential pathways for the impact of positive psychology interventions. They suggest that positive interventions may be effective through their influence on positive emotions, positive behaviors, positive thoughts and need satisfaction. Their hypothesis is that the positive intervention increases one or more of these four mediating variables, which in turn then influence happiness and positive affect as outcomes. The role that may be played by positive emotions in this process is a key one, as we explore next.

\section{Positive emotions as a Key process in positive psychology interventions}

Positive emotions research has been galvanized and inspired by Fredrickson's (1998) seminal broaden-and-build theory of positive emotions. The broaden-and-build model has subsequently led to the development of an understanding of the role, process and outcomes of positive emotions. Indeed, in the last decade or so, researchers have established that positive emotions are an active ingredient in resilience (Fredrickson et al. 2003), increase the experience of happiness and emotional well-being (Fredrickson \& Joiner 2000), speed recovery from negative events (Fredrickson \& Levenson 1998), broaden cognitive repertoires (Fredrickson \& Branigan 2005) - including eliminating the own-face bias in cross-race facial recognition (Johnson \& Fredrickson 2005), and build sustainable positive resources in terms of relationships (e.g., Fredrickson et al. 2008; Waugh \& Fredrickson 2006) as well as psychological 
assets (e.g., Cohn \& Fredrickson 2010; see Fredrickson in press, for an up-to-date review of these extensive findings).

Positive emotions clearly have an important role to play across a range of outcomes, as originally proposed by Fredrickson's (1998) broaden-and-build theory and now borne out by the many studies supporting its key principles. Based on this evidence, it is quite possible that at least one 'active ingredient' in positive psychology interventions is the triggering of positive emotion.

\section{Naturalistic approaches to increasing positive emotions and happiness}

Human beings have been naturally focused on doing things that increase their positive emotion, and have their own naturalistic understanding of what it takes to do so (McMahan \& Estes 2011). For example, in one of the few studies to date to take a naturalistic approach to enhancing happiness, Parks et al. (2012) found that, on average, people performed their chosen happiness increasing strategies several times a week for at least an hour each time. Clearly, then, people are generally inclined towards increasing their levels of positive emotion and happiness, whether this is through the formal practice of positive psychology interventions, or the more informal practice of more everyday, naturalistic activities that people believe will increase their happiness.

One of these naturalistic, everyday activities that typically increases positive emotion and happiness is eating. Eating provides an everyday source of happiness for most people (Macht et al. 2005; Wrzesniewski et al. 2003). Indeed, in free report, people will readily identify eating as an activity that gives them pleasure (Berenbaum 2002). The literature on taste as one of the sensory sources of pleasure (Rozin 1999; Veldhuizen et al. 2010) also suggests happiness may be derived from the taste of consuming specific foods.

People naturally employ everyday strategies to improve their well-being such as the consumption of specific 'mood foods' that are usually highly desirable. Although ice cream is a well-known example of a mood food in the popular media, only a few studies have investigated the effects of ice cream on mood scientifically. Walla et al. (2010) compared the effects of ice cream with those of yoghurt and chocolate. Modulation of the startle response (a sudden involuntary movement in response to an intense and unexpected stimulus) was used as a measure of appetitive motivational state. Particularly in males, the amplitude of the startle response was lower after consumption of ice cream as compared to after consumption of yoghurt or chocolate, indicating that ice cream consumption enhanced the appetitive motivational state.

Another line of evidence stems from an fMRI study by Burger and Stice (2012). Ice cream consumption activated the oral somatosensory brain areas reflecting perception of taste, temperature, and texture more when compared to a tasteless solution. Ice cream also activated brain areas related to reward and motivation, and the magnitude of this activation was positively associated with lower habitual ice cream consumption. This indicates that ice cream is a pleasurable experience when consumed in moderation. However, although perhaps obvious from a consumer point of view, there is no research on whether people actually consciously experience that ice cream makes them happy. Therefore we set out to investigate the effect of eating ice cream on well-being, given its prevalence in popular culture as a 'mood food'. 
In early experimental studies of emotion induction, food was used as a method for increasing positive emotions, most notably Isen and Levin (1972) who gave individuals cookies as a means of inducing positive affect. More recently, Martin et al. (2012) examined the impact of eating dark chocolate, milk chocolate and crackers with cheese spread on anxiety, energy and emotions. They found different effects for anxiety and energy across participants and food types, but no impact on emotions, suggesting that different foods may impact on emotions in different ways. This was one reason why we were interested to investigate the impact of ice cream consumption on affect, and to compare this with a simple gratitude activity.

\section{The current studies: combining and comparing eating and gratefulness activities}

In the current study, we were focused on simple everyday interventions that could be completed quickly and with the minimum of additional props, but which would still be able to demonstrate increases in positive affect. In doing so, this would allow us to combine and compare eating ice cream as a naturalistic mood induction with a simple gratitude activity. A simple gratitude activity appeared to meet both of these criteria.

Within the positive psychology intervention literature, two broad types of gratitude activities have been described: listing things to be grateful for (including 'counting your blessings', (Emmons \& McCullough 2003), as well as grateful contemplation in a more global fashion (Watkins et al. 2003), and behavioral expressions of gratitude (writing a gratitude letter, Seligman et al., 2005)). The gratitude activity often used involves writing a list of things for which one is grateful on a regular basis. Emmons and McCullough (2003) described this activity in experimental settings. Their studies showed that people who write down a list of things they are grateful for on a daily basis for two weeks reported more positive affect and less negative affect compared to people listing daily hassles for two weeks.

In another set of studies, Watkins et al. (2003) described the effects of a single gratitude intervention across two studies. In the first study, students were asked to recall things they did over the previous summer they felt grateful for. The gratitude group reported less negative affect than the control group whereas positive affect was not influenced. In the second study, participants were either asked to think or write about someone to whom they were grateful, or to write a letter to someone whom they were grateful to, or to write about the layout of their living room (neutral condition). All gratitude conditions increased positive affect as compared to the control condition; negative affect was reduced as well but not significantly.

For the current studies, we set out to do two things. First, we combined a simple gratitude activity (writing down three things for which one was grateful that day) with the naturalistic act of eating an ice cream. Our intention in doing so was to tap into the reflective, savoring experience that often accompanies eating an ice cream, by combining this specifically with the reflection required for the gratitude activity. In our second study, we designed an independent-groups study with four groups to explore any differential effects for eating ice cream on its own, completing the gratefulness activity on its own, eating ice cream while completing the gratefulness activity (as per Study 1), together with a control group who undertook a neutral but naturalistic control activity. 


\section{Study 1 \\ Participants}

Participants were 89 undergraduate students from a UK University, of whom 13 were male and 76 were female. The mean age of participants was 20.05 years $(\mathrm{SD}=0.10$ years, range $=19-23$ years). Participants were typically from a Caucasian background (72\%), with other predominant ethnic backgrounds being Indian (16\%) and Black (7\%). Ethical approval was obtained from the University's Ethics Committee.

\section{Design and measures}

There were four days in study 1: two (Monday \& Tuesday) with no activity and two days (Wednesday \& Thursday) with the activity. We chose only two days for the activity because we were focused on simple, brief and naturalistic activities, rather than activities that would be a considerable deviation from people's usual daily lives, as might be the case with more traditional positive psychology interventions.

During these four days, participants recorded levels of positive and negative affect using the 10-item version of the Positive and Negative Affect Scale (PANAS; Watson et al. 1988) twice a day - once in the afternoon and once in the evening - as an indicator of momentary feelings of well-being. During the latter two days of the main part of the study, all participants received a gratefulness activity which they were asked to carry out whilst eating an ice cream.

Participants were instructed: "Please think about three things that you have in your life today and for which you are grateful. Please provide brief details of these below." The page then continued "Today I am grateful / thankful for:" and went on to provide spaces for completion, numbered 1,2 and 3. Participants were instructed to write about these three things for which they were grateful whilst consuming an ice cream (Cornetto Classico ice cream or Cornetto Strawberry ice cream) obtained with a voucher. Participants were free to make their own choice from these ice creams. After this, they completed the PANAS.

At the end of the day, the participants completed the PANAS again. Once completed, these questionnaires were posted in a drop box at the university, which was emptied at the conclusion of each relevant time point. For all study measures, participants also recorded their time of completion using the 24-hour clock. This time was checked to ensure it was accurate for the questionnaire that should have been completed for this time of the study. Participants received a payment of $£ 40$ (c. USD \$60) in compensation for their time at the conclusion of the study.

On separate days before and after the study (the Wednesday of the week before the study and the Wednesday of the week after the study), positive and negative affect were measured with the 20-item version of the PANAS (Watson et al. 1988).

\section{Data analyses}

The data were examined for normality; the negative affect scores were positively skewed and a Box-Cox transformation with $\lambda=-1$ was used to correct this before fitting the ANCOVA. Estimates of the means and standard error terms from this model are shown below back-transformed to the original units. The positive affect and negative affect scores collected on the third and fourth day of the study following the activity were analyzed using a two-way analysis of covariance (ANCOVA) with Day and 
Time-of-Day as within-subjects factors and the pre-study score as covariate. The effect of the activity was obtained by comparing the overall means on the first two days of the study with the overall means on the latter two days of the study. The post-study data collected the Wednesday after the week of the study were analyzed using a one-way ANOVA with Day (pre-study, post-study) as within-subjects factor. For all analyses, only main and interaction effects involving the activity are reported.

\section{Results and discussion}

There was a main effect of the Day for positive affect $(t(88)=2.49, p=.015)$. Positive affect was on average $6.2 \%$ higher on the latter two study days on which the participants received the gratefulness-and-ice-cream activity, both during the afternoon as well as in the evening (average score $13.3 \pm 0.4$; range 5-25) as compared to the preceding two study days (average score $12.5 \pm 0.3$; see Figure 1 ). In addition, there was a main effect of Day for negative affect $(t(88)=3.11, p=.003)$. Negative affect was on average $7.6 \%$ lower on the latter two study days on which the participants received the gratefulness-and-ice-cream activity, both during the afternoon as well as in the evening (average score $6.7 \pm 0.2$; range $5-25$ ) as compared to the preceding two study days (average score $7.2 \pm 0.2$; see Figure 2).

Regarding the pre- and post-study measures, a significant main effect of the combined activity was observed on positive affect $(F(1,88)=34.0, p<.001)$. Positive affect was $17 \%$ higher on the day following the four study days as compared to the pre-study day (see Figure 3).

Study 1 demonstrated that a brief, simple, everyday activity (writing about three things for which participants were grateful whilst consuming an ice cream) was able to change positive and negative affect during the afternoon of the study days, an effect that carried over into the evening (note that there were no interactions with the factor Time of Day). To our knowledge, this is the first study investigating changes in affect during the day as moderated by a naturalistic activity. The effect of the combined activity on positive affect even persisted until a week after the study, suggesting that even

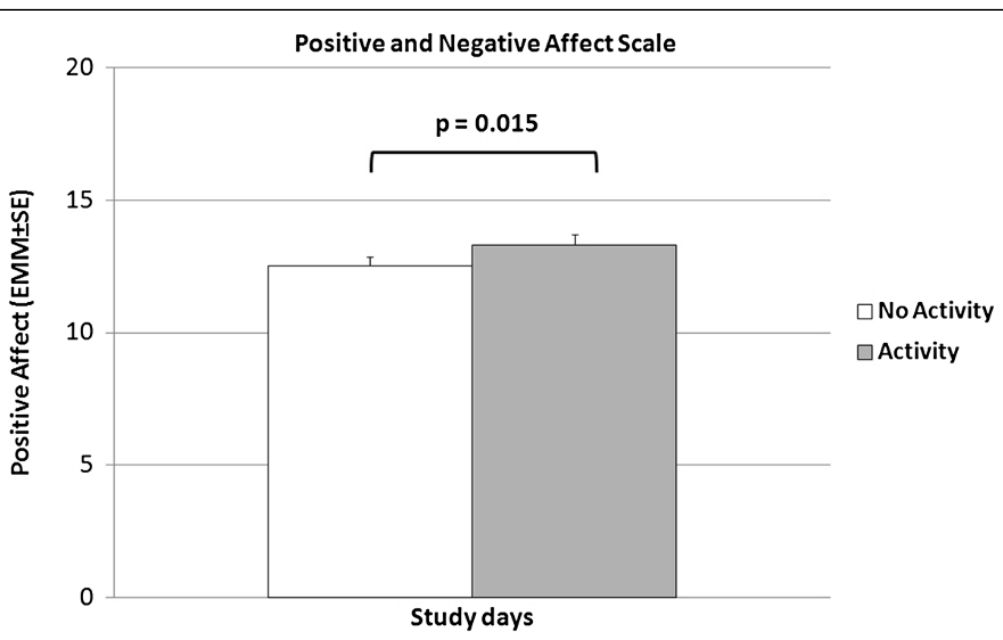

Figure 1 Positive affect on activity and non-activity days. Positive affect was greater on the days during which the participants received the combined gratitude-and-ice-cream activity as compared to the preceding two days (Study 1). 


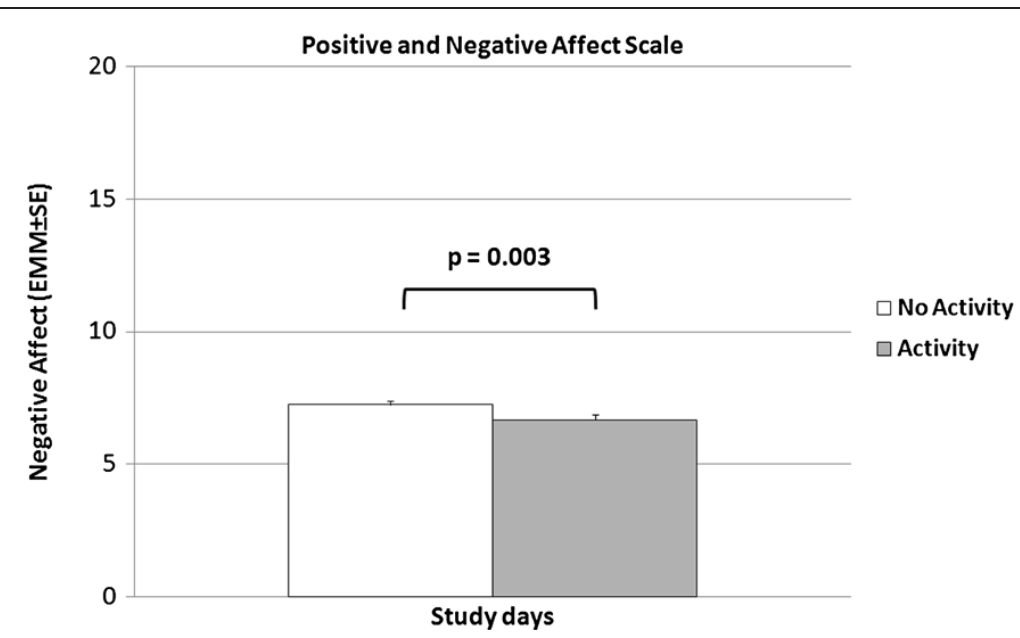

Figure $\mathbf{2}$ Negative affect on activity and non-activity days. Negative affect was lower on the days during which the participants received the combined gratitude-and-ice-cream activity as compared to the preceding two days (Study 1).

simple and short term naturalistic activities may influence positive emotion in a relatively sustainable way.

In Study 1, our focus was on the combination of an everyday eating activity with a simple gratefulness activity, which showed that the two together led to increases in positive affect. Hence, in Study 2 we sought to explore the effects of these two activities both combined once again, as well as independently from each other, together with comparing these to a neutral but naturalistic control activity.

\section{Study 2}

\section{Participants}

Participants were 280 undergraduate students from a UK University, of whom 126 were male and 154 were female. The mean age of participants was 18.95 years

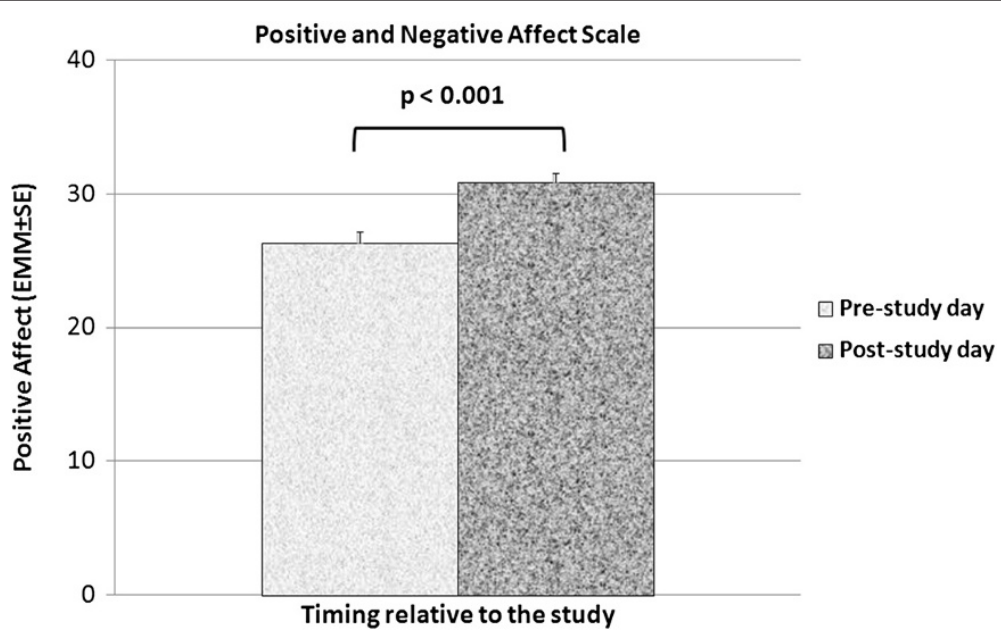

Figure 3 Positive affect on the day before and day after activity days. Positive affect was greater on the post-study day following the four study days as compared to the pre-study days (Study 1). 
$(\mathrm{SD}=1.11$ years, range $=18-26$ years). Participants were typically from a Caucasian background (63\%), with other predominant ethnic backgrounds being Black (13\%) and Indian (10\%). Ethical approval was obtained from the University's Ethics Committee.

\section{Design and measures}

Study 2 had a randomized controlled design with four parallel groups. Group 1 completed the same gratefulness activity as described in the first study (writing about three things for which they were grateful), group 2 consumed an ice cream, group 3 completed the gratefulness activity while consuming an ice cream, and group 4 completed a neutral writing task. The neutral writing task involved writing about that day's weather, serving as a naturalistic control activity, given that the weather is a frequent topic of conversation in this culture. There were two study days in study 2 (Tuesday \& Wednesday) during which the participants received one of the activities above depending on the group to which they were randomly allocated.

During these days, participants recorded levels of positive and negative affect using the 10-item version of the PANAS twice a day - before and after the activity - as an indicator of momentary feelings of well-being. Once completed, the questionnaires were posted in a drop box at the university, which was emptied at the conclusion of each relevant time point. For all study measures, participants also recorded their time of completion using the 24-hour clock. This time was checked to ensure it was accurate for the questionnaire that should have been completed for this time of the study. Participants received a payment of $£ 40$ (c. USD \$60) in compensation for their time at the conclusion of the study.

On separate days before and after the study (the Monday immediately before and the Thursday immediately after the two study days) positive and negative affect were measured with the 20-item version of the PANAS (Watson et al. 1988). Participants also completed the GQ-6 (McCullough et al. 2002) to allow consideration of any changes in their gratitude level as a result of the activities.

\section{Data analyses}

The data were analyzed using 2-way and 3-way analyses of covariance (ANCOVA). The data were examined for normality and kurtosis and deemed acceptable for parametric analyses. The positive affect and negative affect scores collected after the activity on the two study days were analyzed using a 3-way ANCOVA with Day as a within-subjects factor, ice cream and gratefulness activity as between-subjects factors. The affect scores collected before the activity on the two study days were used as a covariate to control for pre-activity levels of positive affect and negative affect. The post-study data collected on day 4 were analyzed using a 2-way ANCOVA with ice cream and gratefulness activity as between-subjects factors and the corresponding baseline measure collected on day 1 as the covariate.

In order to explore the data further, affect balance scores were calculated by subtracting negative affect from positive affect for each of the pre and post data collected on the two study days. The affect balance scores collected after the activity on the two study days were then analyzed using a 3-way ANCOVA with Day as a withinsubjects factor, ice cream and gratefulness activity as between-subjects factors. The 
affect balance scores collected before the activity on the two study days were used as a covariate to control for pre-activity levels of affect.

In addition, to allow consideration of the positive to negative emotion ratios highlighted by Fredrickson and Losada (2005), emotion ratio scores were calculated by dividing positive affect by negative affect for each of the pre and post data collected on the two study days. The emotion ratio scores collected after the activity on the two study days were then analyzed using a 3-way ANCOVA with Day as a within-subjects factor, ice cream and gratefulness activity as between-subjects factors. The emotion ratio scores collected before the activity on the two study days were used as a covariate to control for pre-activity emotion ratios. Only main and interaction effects involving either activity are reported.

\section{Results and discussion}

On the activity days (Days 2 and 3), significant main effects of gratefulness activity $\left(F(1,274)=10.5, p=.001\right.$, partial $\left.\eta^{2}=.037\right)$ and ice cream $(F(1,274)=8.4, p=.004$, partial $\left.\eta^{2}=.030\right)$ were observed for positive affect. Positive affect was on average $22 \%$ greater in the gratefulness-activity and ice cream groups (average score $14.7 \pm 0.4$; range 5-25) as compared to the control group (average score $12.0 \pm 0.4$; see Figure 4). The interaction between ice cream and gratefulness activity was also significant $\left(F(1,274)=11.8, p=.001\right.$, partial $\left.\eta^{2}=.041\right)$, indicating that the increase in positive affect was similar to that following either the gratefulness activity or eating ice cream, and that the effects of the two activities were not additive. There were no main or interaction effects involving the factor Day, indicating that the effects were similar on both activity days.

For negative affect on the activity days (on average $8.7 \pm 0.3$; range 5-25), main and interaction effects of ice cream and gratefulness activity were not significant (all $F_{\mathrm{s}}<0.5$; all $p \mathrm{~s}>.48$; see Figure 5), indicating that neither activity influenced negative affect.

On the post-study day (Day 4), a significant main effect of the gratefulness activity was observed on self-reported gratefulness as measured with the GQ-6 $(F(1,275)=14.3$,

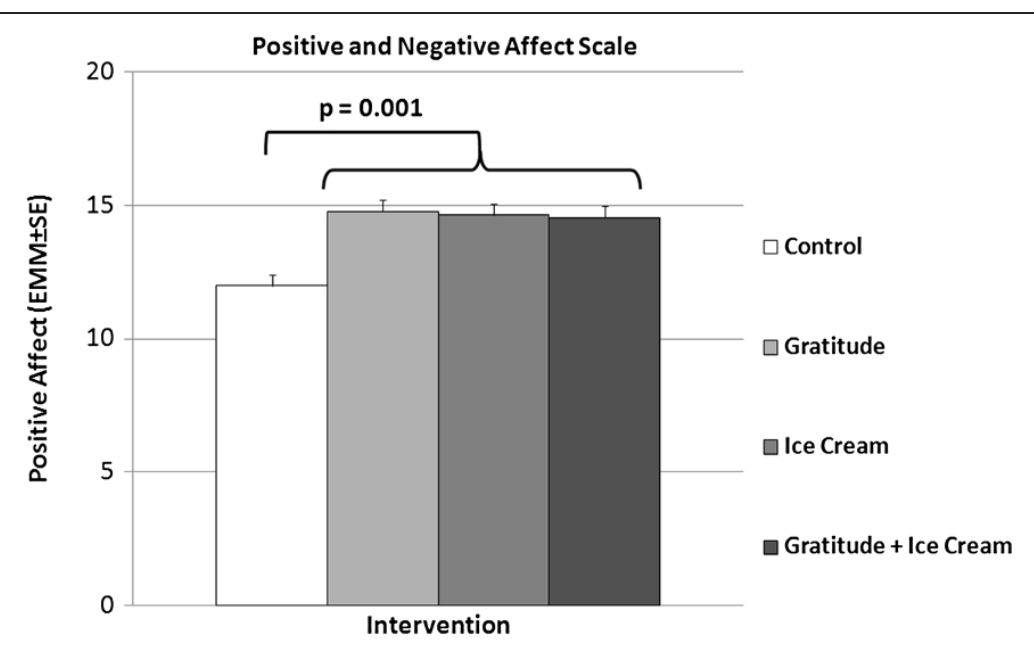

Figure 4 Positive affect following activity days. Positive affect (corrected for baseline) was greater in the groups that had received the gratitude and / or ice cream activity as compared to the control activity (Study 2). 


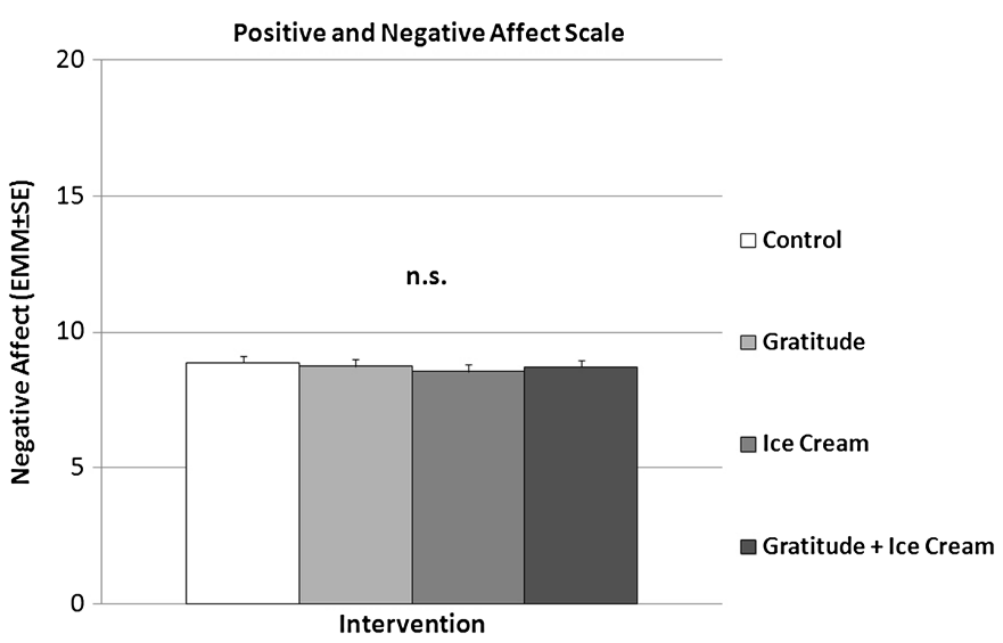

Figure 5 Negative affect following activity days. Negative affect (corrected for baseline) did not differ between groups, indicating that neither activity had an influence on negative affect (Study 2).

$p<.001$, partial $\eta^{2}=.049$ ). Self-reported gratefulness was $5 \%$ higher in the groups that had received the gratefulness activity on the two previous days as compared to the other groups (see Figure 6).

On the activity days (Days 2 and 3), significant main effects of gratefulness activity $\left(F(1,274)=7.5, p=.007\right.$, partial $\left.\eta^{2}=.027\right)$ and ice cream $(F(1,274)=7.9, p=.005$, partial $\left.\eta^{2}=.028\right)$ were observed for affect balance, indicating higher levels of affect balance (that is, higher positive affect) in the gratefulness-activity and ice cream groups. The interaction between ice cream and gratefulness activity was also significant $\left(F(1,274)=10.1, p=.002\right.$, partial $\left.\eta^{2}=.035\right)$, indicating that the increase in affect balance was similar to that following either the gratefulness activity or eating ice cream, and that the effects of the two activities were not additive. There were no main or interaction effects involving the factor Day, indicating that the effects were similar on both activity days.

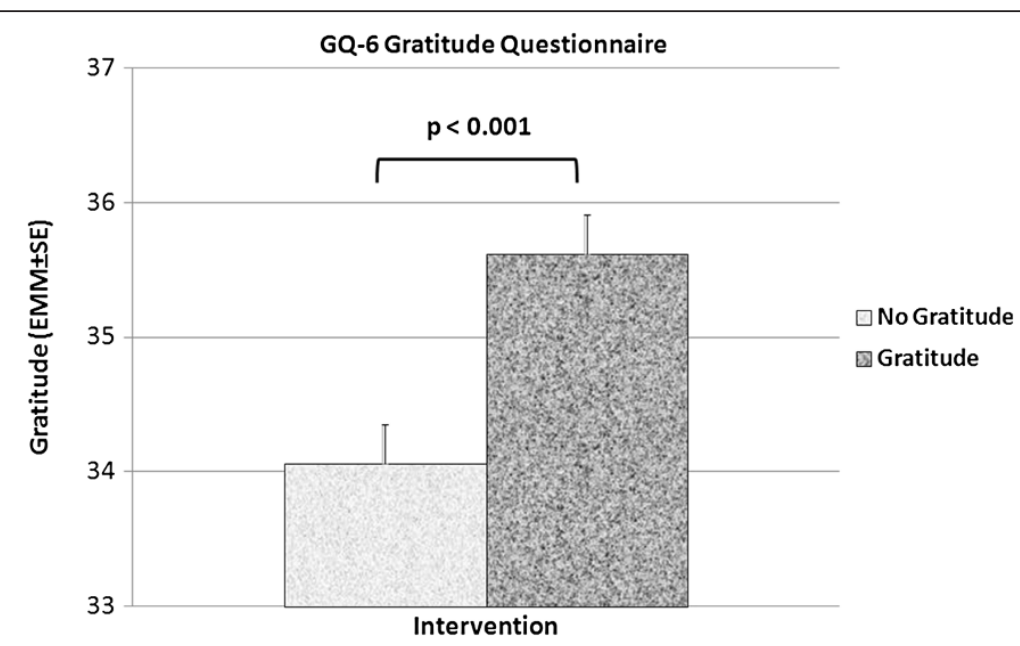

Figure 6 Gratitude levels after the activity days. Self-reported gratitude (corrected for pre-activity level) was greater in the groups that had received the gratitude activity as compared to the other groups (the ice cream activity had no effect) (Study 2). 
On the activity days (Days 2 and 3), significant main effects of gratefulness activity $\left(F(1,274)=6.5, p=.012\right.$, partial $\left.\eta^{2}=.023\right)$ and ice cream $(F(1,274)=8.9, p=.003$, partial $\left.\eta^{2}=.031\right)$ were observed for emotion ratio, indicating higher emotion ratios (that is, higher levels of positive emotion relative to negative emotion) in the gratefulness-activity and ice cream groups. The interaction between ice cream and gratefulness activity was also significant $\left(F(1,274)=7.0, p=.009\right.$, partial $\left.\eta^{2}=.025\right)$, indicating that the increase in emotion ratios was similar to that following either the gratefulness activity or eating ice cream, and that the effects of the two activities were not additive. There were no main or interaction effects involving the factor Day, indicating that the effects were similar on both activity days.

\section{General discussion}

Across two studies, results showed that completing the simple, naturalistic activities of writing down three things for which one is grateful and eating an ice cream led to significant increases in positive affect. In study 1 , we found this effect for the combined activity of eating an ice cream while writing down three things for which one was grateful/thankful. In study 2, we extended this research design to include three experimental groups, separating out the gratefulness activity from the ice cream consumption, as well as again combining the two together, and comparing all three against a neutral but naturalistic control condition of writing about the weather (a popular topic of conversation). For each experimental group, we found that positive affect increased - although notably there was no additive effect for combining the gratefulness activity with eating ice cream - results for this were comparable to doing either one on its own.

Whereas other studies have shown the effects on positive affect of more sustained gratitude activities (e.g., Emmons \& McCullough 2003; Watkins et al. 2003), in this study we were more interested to take a more naturalistic, everyday approach to examining the impact on positive affect of more typical activities that people may undertake. We chose eating an ice cream as there is some evidence to suggest that eating ice cream in moderation increases mood (Burger \& Stice 2012; Walla et al., 2010) and also because ice cream is recognized as a typical 'mood food' in popular culture. We chose the gratefulness activity because it was very simple and very short, allowing it to be completed with the minimum of additional props, in the minimum time, while simultaneously eating an ice cream. This allowed us to tap into the opportunity for reflection and savoring that this ice cream consumption may allow, and which again is part of its image in popular culture as a 'mood food', while considering this both separately and together with the gratefulness activity.

Our results also showed that the gratefulness activity had a significant effect on selfreported gratitude, with scores on the GQ-6 increasing significantly for the group who had undertaken the gratefulness activity. This suggests that the gratefulness activity specifically not only increased positive affect, it also increased gratitude, a finding which has interesting implications for the underlying theory of how positive psychology interventions may operate.

Layous and Lyubomirsky (in press) have proposed that positive psychology interventions may work through four mediating pathways: positive emotions, positive thoughts, positive behaviors, and need satisfaction. Our findings suggest that the gratefulness activity that we employed led to both increased positive affect (the positive emotions 
mediating pathway) and also positive thoughts (increased gratitude and gratefulness). While the current study cannot attest this directly, one might hypothesize that these increases in positive emotions and positive thoughts may also lead to enhanced positive behaviors (e.g., being more grateful and prosocial towards others). Taken together, these positive mediating pathways may then lead to longer term positive outcomes, such as the increased happiness that is found in positive psychology interventions that are maintained for an extended period of time (e.g., Seligman et al. 2005, reported that their 6-month effects were fully explained by whether the participant continued with the activity after the 1-week intervention period).

This study also speaks to the role of positive emotions in everyday life, and specifically the positive emotions that may be triggered by naturalistic, everyday activities. Whereas positive psychology interventions have tended to be relatively non-natural (that is, inviting people to undertake activities they would not normally do, such as writing a gratitude letter, performing a random act of kindness, or writing about their best possible self), our focus in the current studies was to be more naturalistic. Hence, participants consumed an ice cream of their choice in a setting of their choosing, while reflecting on and noting down three things for which they were grateful (the one slightly less natural element of our activity, but which nonetheless was very simple and short to complete). Our results suggest that even simple, everyday, naturalistic activities such as this can reliably influence and increase positive affect.

Given that these simple activities are things that people may do every day of their own accord, our findings speak to the potential role of normal, everyday activities in generating small amounts of positive emotion that may nonetheless accumulate over time and influence longer term happiness and well-being. With the extensive research base that now exists for the importance and impact of positive emotions (see Fredrickson in press, for review), research attention should also take account of how people generate positive emotions for themselves through the small things they do as part of everyday life. This is especially important when one recognizes that we found the same pattern of results for positive affect when comparing results for affect balance (the balance between positive affect and negative affect) and also the ratio between positive and negative emotions. Fredrickson and Losada (2005) showed that for individuals to flourish, they needed to experience positive to negative emotion ratios in excess of 3:1. Our studies show that even simple everyday activities can be one route to achieving better affect balance and an improved ratio of positive to negative emotions.

Of course, this study is not without its limitations, and future research is recommended to address some of these, as well as exploring new questions which have arisen as a result. First, notwithstanding ice cream's position in the popular culture as a 'mood food', future studies could monitor whether the initial positive impact of eating ice cream is sustained over time or gives way to negative emotions later. The inclusion of implicit measures of mood, or physiological indicators, would help to address this. Further, it is possible that demand characteristics of the study meant that people consumed ice cream when they would not otherwise have chosen to do so. Future research might consider settings that are even more naturalistic than we managed to achieve here, in order to address this.

Second, while researchers have considered the psychological mechanisms through which everyday activities or indeed positive psychology interventions may impact on 
positive emotions and happiness, there is also a need to explore the neural pathways and brain structures that may be operational in the neuroscience of these processes (e.g., Berridge \& Kringelbach 2011).

Third, while we recorded participants' time of completion on their questionnaires and checked these against the relevant times for the emptying of the drop box, there remains the possibility that these may have been inaccurate, or that, despite using a voucher to receive a free ice cream, participants may not have consumed it. Future studies may wish to address this by using smartphones or palm pilots to record this information in real time.

Fourth, there is an opportunity for research to focus on more of the everyday, typical and naturalistic experiences of people, and the particular decisions that people make at numerous points throughout each day, that are designed, implicitly or explicitly, to enhance their well-being (McMahan \& Estes 2011). Research into positive psychology interventions to date has tended to focus on particular interventions that have been developed by researchers, rather than a more naturalistic consideration of people's everyday activities that would allow a battery of simple happiness-enhancing activities to be built 'from the ground up'. Positive psychology and the advent of positive psychology interventions research have undoubtedly increased the sum total of our knowledge about human happiness, but it would be a huge missed opportunity if, in doing so, researchers lost sight of the everyday routes to happiness that people are using according to their own naturalistic, implicit theories and experiences.

Abbreviations

ANCOVA: Analysis of covariance; GQ-6: Gratitude questionnaire - 6; PANAS: Positive and negative affect scale.

Competing interests

$\mathrm{EdB}, \mathrm{CT}, \mathrm{RH}$ \& JW are all employed by Unilever which owns a range of ice cream brands.

\section{Authors' contributions}

All authors contributed to the design and analysis of the studies. Study implementation was led by JM. Writing of the paper was led by PAL. All authors read and approved the final manuscript.

\section{Acknowledgements}

We are grateful to Peter Murray for his statistical support for the first study, and to Sella Masselink for their assistance with running the second study. The studies were funded by Unilever (www.unilever.com).

\section{Author details}

${ }^{1}$ Centre of Applied Positive Psychology, Coventry, UK. ' Unilever R\&D, Vlaardingen, The Netherlands. ${ }^{3}$ Unilever Research, Colworth Science Park, MK44 1LQ Bedford, UK. ${ }^{4}$ University of Leicester, Leicester, UK.

Received: 5 October 2012 Accepted: 2 October 2013

Published: 12 Nov 2013

References

Berenbaum, H. (2002). Varieties of joy-related pleasurable activities and feelings. Cognition and Emotion, 16(4), 473-494. Berridge, KC, \& Kringelbach, ML. (2011). Building a neuroscience of pleasure and well-being. Psychology of Well-Being: Theory, Research and Practice, 1, 3. doi:10.1186/2211-1522-1-3.

Boehm, JK, Lyubomirsky, S, \& Sheldon, KM. (2011). A longitudinal experimental study comparing the effectiveness of happiness-enhancing strategies in Anglo Americans and Asian Americans. Cognition and Emotion, 25, 1152-1167.

Brickman, P, \& Campbell, DT. (1971). Hedonic relativism and planning the good society. In MH Appley (Ed.), Adaptation-level theory (pp. 287-302). New York: Academic Press.

Burger, KS, \& Stice, E. (2012). Frequent ice cream consumption is associated with reduced striatal response to receipt of an ice cream-based milkshake. American Journal of Clinical Nutrition, 95, 810-817.

Burton, CM, \& King, LA. (2004). The health benefits of writing about intensely positive experiences. Journal of Research in Personality, 38, 150-163.

Cohn, MA, \& Fredrickson, BF. (2010). In search of durable positive psychology interventions: predictors and consequences of long-term behavioral change. Journal of Positive Psychology, 5, 355-366.

Diener, E, Lucas, RE, \& Scollon, CN. (2006). Beyond the hedonic treadmill: revising the adaptation theory of well-being. American Psychologist, 61, 305-314. 
Emmons, RA, \& McCullough, ME. (2003). Counting blessings versus burdens: an experimental investigation of gratitude and subjective well-being in daily life. J Pers Soc Psychol, 84, 377-389.

Fordyce, MW. (1977). Development of a program to increase personal happiness. J Couns Psychol, 24, 511-521.

Fredrickson, BL. (1998). What good are positive emotions? Review of General Psychology, 2(3), 300-319.

Fredrickson, BL. (in press). Positive emotions broaden and build. In EA Plant \& PG Devine (Eds.), Advances in experimental social psychology (Vol. 49). San Diego, CA: Academic Press.

Fredrickson, BL, \& Branigan, C. (2005). Positive emotions broaden the scope of attention and thought-action repertoires. Cognition and Emotion, 19, 313-332.

Fredrickson, BL, Cohn, MA, Coffey, KA, Pek, J, \& Finkel, SM. (2008). Open hearts build lives: positive emotions, induced through loving-kindness meditation, build consequential personal resources. J Pers Soc Psychol, 95(5), 1045-1062.

Fredrickson, BL, \& Joiner, T. (2000). Positive emotions trigger upward spirals toward emotional wellbeing. Psychol Sci, $13,172-175$.

Fredrickson, BL, \& Levenson, RW. (1998). Positive emotions speed recovery from the cardiovascular sequelae of negative emotions. Cognition and Emotion, 12, 191-220.

Fredrickson, BL, \& Losada, MF. (2005). Positive affect and the complex dynamics of human flourishing. American Psychologist, 60, 678-686.

Fredrickson, BL, Tugade, MM, Waugh, CE, \& Larkin, GR. (2003). What good are positive emotions in crises? a prospective study of resilience and emotions following the terrorist attacks on the United States on September $11^{\text {th }}, 2001$. J Pers Soc Psychol, 84(2), 365-376.

Isen, AM, \& Levin, PF. (1972). The effect of feeling good on helping: cookies and kindness. J Pers Soc Psychol, 21, 384-388.

Johnson, KJ, \& Fredrickson, BL. (2005). "We all look the same to me:" positive emotions eliminate the own-race bias in face recognition. Psychol Sci, 16, 875-881.

Layous, K, \& Lyubomirsky, S. (in press). The how, who, what, when, and why of happiness: mechanisms underlying the success of positive interventions. In J Gruber \& J Moscowitz (Eds.), The light and dark side of positive emotions. New York: Oxford University Press.

Lyubomirsky, S, Dickerhoof, R, Boehm, JK, \& Sheldon, KM. (2011). Becoming happier takes both a will and a proper way: an experimental longitudinal intervention to boost well-being. Emotion, 11, 391-402.

Macht, M, Meininger, J, \& Roth, J. (2005). The pleasures of eating: a qualitative analysis. Journal of Happiness Studies, 6, 137-160.

Martin, F-PJ, Antille, N, Rezzi, S, \& Kochhar, S. (2012). Everyday eating experiences of chocolate and non-chocolate snacks impact postprandial anxiety, energy and emotional states. Nutrients, 4, 554-567.

McCullough, ME, Emmons, RA, \& Tsang, J-A. (2002). The grateful disposition: a conceptual and empirical topography. $J$ Pers Soc Psychol, 82, 112-127.

McMahan, EA, \& Estes, D. (2011). Measuring lay conceptions of well-being: the beliefs about well-being scale. Journal of Happiness Studies, 12, 267-287.

Parks, AC, Della Porta, MD, Pierce, RS, Zilca, R, \& Lyubomirsky, S. (2012). Pursuing happiness in everyday life: a naturalistic investigation of online happiness seekers. Emotion, 12(6), 1222-1234.

Rozin, P. (1999). Preadaptation and the puzzles and properties of pleasure. In D Kahneman, E Diener, \& N Schwarz (Eds.), Well-being: the foundations of hedonic psychology (pp. 109-132). New York: Russell Sage Foundation.

Seligman, MEP, Steen, TA, Park, N, \& Peterson, C. (2005). Positive psychology progress: empirical validation of interventions. American Psychologist, 60, 410-421.

Sheldon, KM, Boehm, JK, \& Lyubomirsky, S. (in press). Variety is the spice of happiness: the hedonic adaptation prevention (HAP) model. In I Boniwell \& S David (Eds.), Oxford handbook of happiness. Oxford: Oxford University Press.

Sheldon, KM, \& Lyubomirsky, S. (2006). Achieving sustainable gains in happiness: change your actions, not your circumstances. Journal of Happiness Studies, 7, 55-86.

Sin, NL, \& Lyubomirsky, S. (2009). Enhancing well-being and alleviating depressive symptoms with positive psychology interventions: a practice-friendly meta-analysis. Journal of Clinical Psychology: In Session, 65, 467-487.

Veldhuizen, MG, Rudenga, KJ, \& Small, DM. (2010). The pleasure of taste, flavor and food. In ML Kringelbach \& KC Berridge (Eds.), Pleasures of the brain (pp. 146-168). New York: Oxford University Press.

Walla, P, Richter, M, Farber, S, Leodolter, U, \& Bauer, H. (2010). Food-evoked changes in humans' startle response modulation and event-related brain potentials (ERPs). Journal of Psychophysiology, 24, 25-32.

Watkins, PC, Woodward, K, Stone, T, \& Kolts, RL. (2003). Gratitude and happiness: development of a measure of gratitude, and relationships with subjective well-being. Social Behavior and Personality, 31, 431-451.

Watson, D, Clark, LA, \& Tellegen, A. (1988). Development and validation of brief measures of positive and negative affect - the PANAS scales. J Pers Soc Psychol, 54, 1063-1070.

Waugh, CE, \& Fredrickson, BL. (2006). Nice to know you: positive emotions, self-other overlap, and complex understanding in the formation of new relationships. Journal of Positive Psychology, 1, 93-106.

Wrzesniewski, A, Rozin, P, \& Bennett, G. (2003). Working, playing, and eating: making the most of most moments. In CLM Keyes \& J Haidt (Eds.), Flourishing: positive psychology and the life well-lived (pp. 185-204). Washington, D.C.: American Psychological Association.

10.1186/2211-1522-3-6

Cite this article as: Linley et al:: Two simple, brief, naturalistic activities and their impact on positive affect: feeling grateful and eating ice cream. Psychology of Well-Being: Theory, Research and Practice 2013, 3:6 